\title{
Diet quality as a predictor of healthy and cardiometabolic disease-free life expectancy between ages 50 to 85
}

\author{
$\underline{\text { Hanna Lagström }}^{1,2}$, Sari Stenholm ${ }^{1,2}$, Tasnime Akbararly ${ }^{3,4}$, Jaana Pentti ${ }^{1,2}$, Jussi Vahtera ${ }^{1,2}$, \\ Mika Kivimäki ${ }^{5,6}$ and Jenny Head ${ }^{5}$ \\ ${ }^{1}$ Department of Public Health, University of Turku, Turku, Finland, \\ ${ }^{2}$ Centre for Population Health Research, University of Turku, Turku, Finland, \\ ${ }^{3}$ Inserm, U1198, Université Montpellier, Ecole Pratique des Hautes Etudes, Montpellier, France, \\ ${ }^{4}$ Department of Psychiatry \& Autism Resources Centre, University Research and Hospital Center of Montpellier, \\ Montpellier, France, \\ ${ }^{5}$ Department of Epidemiology and Public Health, University College London, London, United Kingdom and \\ ${ }^{6}$ Clinicum, Faculty of Medicine, University of Helsinki, Helsinki, Finland
}

\begin{abstract}
Poor dietary quality is one of the leading modifiable risk factor for premature mortality worldwide. People live longer than ever, but spend more years with illness and disability although the ultimate goal is to increase healthy years of life. Less research has considered the role of dietary habits in relation to health or cardiometabolic disesase-free life expectancy (LE). This study investigate the association of diet quality with healthy and cardiometabolic disease-free LE between ages 50 and 85 . The study comprised 8,075 participants of the Whitehall II study. Diet quality was assessed with Alternative Healthy Eating Index (AHEI) 2010 at phases 3,5 and 7 and took the measure of diet closest to age of 50 years for each participant. We utilized repeat measures of self-rated health and cardiometabolic disease from the first observation when participants were aged 50 years or older. In the analyses the AHEI-2010 total score was categorized in to quintiles, where the lowest quintile represents unhealthiest diet quality and highest quintiles healthiest diet. Multistate life table models were used to estimate healthy and cardiometabolic disease-free LE from age 50 to 85 years for each category of AHEI-2010 quintiles and three occupational position group. Participants in the highest AHEI-2010 quintile lived 3.6 years longer in good health and 2.7 years longer without cardiometabolic diseases than participants in the lowest quintile of the AHEI-2010. Higher diet quality associated with an increased healthy and cardiometabolic disease-free LE was observed across different occupational positions: Men in highest occupational position and highest AHEI-2010 quintile lived 9.4 years longer with good health compared to lowest occupational position and lowest AHEI-2010 quintile and for women the corresponding difference was 8.2 years. In terms of proportion of years spent without cardiometabolic diseases ranged from $77 \%$ (high occupational position and highest AHEI-2010) to 57\% (low occupational position and lowest AHEI-2010) in men and from 82\% to 70\% in women. The difference in years lived healthy across AHEI-2010 quintiles was most remarkable in persons with low occupation status. Healthier dietary habits are associated with longer healthy and longer cardiometabolic disease-free LE between ages 50 and 85 . Attention to poor diet should be paid especially among people in the lowest occupational position.
\end{abstract}

\section{Conflict of Interest}

There is no conflict of interest. 